\title{
Association of metabolic syndrome with TyG index and TyG- related parameters in an urban Chinese population: a 15-year prospective study
}

Xin Zhang

West China School of Medicine: Sichuan University West China Hospital

Shanshan Jia

West China School of Medicine: Sichuan University West China Hospital

zhipeng zhang

West China School of Medicine: Sichuan University West China Hospital

runyu ye

West China School of Medicine: Sichuan University West China Hospital

xiangyu yang

West China School of Medicine: Sichuan University West China Hospital

xiaoping chen ( $\nabla$ chenxphtn@163.com )

West China School of Medicine: Sichuan University West China Hospital https://orcid.org/0000-0003-4582-2051

\section{Research}

Keywords: Metabolic syndrome, TyG index, TyG-related parameters, Obesity markers, Chinese population

Posted Date: February 25th, 2022

DOI: https://doi.org/10.21203/rs.3.rs-1375029/v1

License: (c) (i) This work is licensed under a Creative Commons Attribution 4.0 International License. Read Full License 


\section{Abstract}

Background: The metabolic syndrome(Mets) is a multiplex risk factor for atherosclerotic cardiovascular diseases $₫ T$ The aims of the study were to assess the association of the Mets with TyG index and TyG-related parameters in an urban Chinese population.

Methods and Results: The data were collected in 1992 and then again in 2007 from the same group of 590 individuals (363 males and 227 females ) without Mets in 1992. During 15 years' follow-up, TyG-waist to height ratio (TyG-WHtR) shows the largest AUC for MS detection (0.686) followed by TyG-waist circumference (TyG-WC) (0.660), TyG-waist-to-hip ratio (TyGWHpR) (0.564), and TyG index (0.556) in all participants. Gender analysis revealed that TyG-WHtR and TyG-WC have the largest AUC in both genders. TyG-WHtR significantly predicted Mets in all participants, with an unadjusted odds ratio of 5.63 (95\% Cl: 3.23-9.83 Pख0.001). Associations remained significant after adjustment for smoking, drinking, physical exercise and components of Mets.

Conclusions: TyG-WHtR might be a strong and independent predictor for Mets in all participants in an urban Chinese population. TyG-related markers that combine obesity markers with TyG index are superior to other parameters in identifying Mets in both genders.

\section{Introduction}

Metabolic syndrome (Mets) is a cluster of metabolic abnormalities characterized by abdominal obesity, hypertension, dyslipidemia, abnormal glucose metabolism, or previously diagnosed type 2 diabetes[1]. Lipid metabolism disorder is a common metabolic abnormality in patients with Mets, and closely related to atherosclerosis, significantly increased the risk of cardiovascular diseases[2]. Insulin resistance (IR) is characterized by impaired tissue sensitivity or responsiveness to circulating insulin, which plays an important role in the development of Mets[3]. The triglycerides and glucose (TyG) index combine fasting plasma glucose (FPG) and fasting triglycerides (TG), is a novel tool that has been suggested to help as a surrogate marker for IR[4]. Many evidence has shown that there was a strong correlation between the TyG index and type 2 diabetes mellitus, hypertension, cardiovascular events and fatty liver both in China and elsewhere[5-8].

In recent years, researchers have focused on TyG-related parameters such as product of TyG and waist circumference (TyGWC), TyG and waist-to height ratio (TyG-WHtR), TyG and waist-to-hip ratio (TyG-WHpR) as well as their ability to predict the risk of cardiovascular events[9]. In a cross study, Taiwo $\mathrm{H}$ et al. found that the TyG-related parameters improved identification and prediction of Mets in Nigerians[10]. However, there is no prospective study to explore the relationship between TyG-related parameters and Mets in an urban Chinese population. This study was therefore aimed to prospectively determine the predictive value of TyG-related parameters for the Mets in an urban Chinese population.

\section{Methods}

\section{Study population}

The study population was obtained from a Chinese Multiprovincial Cohort Study (CMCS) in an urban community located in Chengdu, Sichuan province, China. A baseline examination was conducted on 711 participants in 1992 using a risk factor survey developed by the World Health Organization-Multinational Monitoring of Trends and Determinants in Cardiovascular Diseases (WHO-MONICA) [11]. The data were again collected in 2007 from the same group. Detailed information of these participants has been already reported[12-15]. In 1992, each patient's history of hypertension, diabetes mellitus, hyperlipidemia, heart diseases (coronary artery disease, heart failure or arrhythmia), current smoking and current alcohol consumption, as well as their physical exercise habits, was determined by self-administered questionnaires and confirmed by a physician's interview. After at least 5-min of rest in seated position, blood pressure (BP) was measured in the sitting position twice at 2-min interval using an upright standard sphygmomanometer. Waist, height, weight, and body mass index

Page $2 / 12$ 
(BMI) were measured. BMI was calculated as body weight $(\mathrm{kg})$ divided by the height squared $(\mathrm{m} 2)$. Blood was drawn from the antecubital vein in the morning after a 12-h fast for determinations of fasting plasma glucose (FPG), fasting serum TC, LDL-C, HDL-C, and TG. These chemistries were measured at West China Hospital laboratory. Since 114 participants were diagnosed with Mets, 7 participants with heart disease in 1992, they were excluded from the analysis. Therefore, only 590 participants with complete data were available and analysed. This study was approved by the Ministry of Health of China, as well as by the Ethics Committee of West China Hospital of Sichuan University. All participants provided written informed consent. In 2007, we repeated those measurements with the same methods.

\section{Related Definitions}

Mets was defined as the new joint interim statement[1], and the presence of any 3 of 5 after mentioned risk factors constituted a diagnosis of MetS: (1) elevated TG was defined as $1.7 \mathrm{mmol} / \mathrm{L}$ or greater; (2) BP was defined as systolic BP $(\mathrm{SBP}) \geq 130$ and/or diastolic BP (DBP) $\geq 85 \mathrm{mmHg}$ and/or those receiving antihypertensive medications;(3) reduced HDL-C was defined as a level less than $1.0 \mathrm{mmol} / \mathrm{L}$ for males and a level less than $1.3 \mathrm{mmol} / \mathrm{L}$ for females; (4) elevated FPG was defined as $5.6 \mathrm{mmol} / \mathrm{L}$ or greater; (5) for Asians, elevated WC was defined as $80 \mathrm{~cm}$ or greater for females and $90 \mathrm{~cm}$ or greater for males[1, 16]. Smoking: average cigarette consumption $\geq$ one/day. Alcohol intake: average intake of alcohol $\geq 50$ g/day. Physical activity: exercise one or more times per week, at least 20 minutes each time.

\section{Statistical analysis}

Data are presented as the mean \pm SD for normally continuous variables and as frequency (\%) for categorical variables by gender. Additionally, to explore the relationship between TyG index and TyG-related parameters and risk of Mets, both univariate and multivariate logistic regression analyses were used to estimate the odds ratios (ORs) and $95 \% \mathrm{Cl}$ values. Similarly, the ORs and $95 \%$ Cls for the risk of Mets in various parameters across each subgroup were estimated and their interactions were tested. The diagnostic ability of TyG index and TyG index-related parameters to identify people with Mets (as per the harmonized criteria) was determined with the receiver operating characteristic (ROC) curves. Pairwise comparisons between area under the curve (AUC)s for the four parameters were performed. A 2-tailed $p<0.05$ was considered significant in all analysis. All analyses were performed using Empower (R) (http://www.empowerstats. com, X\&Y solutions, Inc., Boston MA) and R (http://www.R-project.org).

\section{Results}

\section{Baseline characteristics of Mets patients and controls}

Table 1 shows the baseline characteristics of the involved population classified by genders. A total of 590 subjects were included in our study, including 363 (61.68\%) males and 227 (38.32\%) females. The mean age of males was older than that of females. The males had higher SBP, DBP, High, Weight, Waist circumference, waist hip rate(WHpR) as well as rate of smoking and alcohol intake. Compared with females, males had higher levels of TG. By contrast, the level of HDL-C was lower in the males. Values of TyG index, TyG-WC and TyG-WHpR were higher in the males than in the females. 
Table 1

Baseline characteristics of the involved population classified by genders.

\begin{tabular}{|llll|}
\hline & Males & Females & P-value \\
\hline N & 363 & 227 & \\
\hline Age & $48.87 \pm 5.73$ & $46.00 \pm 6.13$ & $<0.001$ \\
\hline EH & $41(11.29 \%)$ & $18(7.93 \%)$ & 0.185 \\
\hline FBG(mmol/l) & $4.26 \pm 0.74$ & $4.31 \pm 1.12$ & 0.964 \\
\hline Height(cm) & $165.16 \pm 5.71$ & $154.59 \pm 5.45$ & $<0.001$ \\
\hline Weight(cm) & $62.59 \pm 8.01$ & $54.93 \pm 6.74$ & $<0.001$ \\
\hline Waist(cm) & $77.42 \pm 7.41$ & $72.19 \pm 6.32$ & $<0.001$ \\
\hline Hip(cm) & $91.18 \pm 5.37$ & $91.66 \pm 5.27$ & 0.266 \\
\hline WHpR & $0.85 \pm 0.06$ & $0.79 \pm 0.05$ & $<0.001$ \\
\hline WHtR & $0.47 \pm 0.05$ & $0.47 \pm 0.04$ & 0.649 \\
\hline BMI & $22.94 \pm 2.72$ & $22.97 \pm 2.40$ & 0.825 \\
\hline SBP (mmHg) & $113.40 \pm 12.82$ & $109.91 \pm 12.97$ & 0.001 \\
\hline DBP (mmHg) & $73.15 \pm 8.46$ & $71.34 \pm 8.30$ & 0.011 \\
\hline TG $\llbracket m m o l / L 】$ & $2.04 \pm 0.86$ & $1.86 \pm 0.73$ & 0.011 \\
\hline TC (mmol/L) & $4.42 \pm 0.70$ & $4.50 \pm 0.80$ & 0.294 \\
\hline HDL-C(mmol/L) & $1.24 \pm 0.22$ & $1.30 \pm 0.24$ & $<0.001$ \\
\hline LDL-C(mmol/L) & $2.23 \pm 0.76$ & $2.32 \pm 0.81$ & 0.327 \\
\hline TyG index & $8.76 \pm 0.39$ & $8.68 \pm 0.35$ & 0.020 \\
\hline TyG-WC & $555.38 \pm 67.19$ & $512.30 \pm 55.37$ & $<0.001$ \\
\hline TyG-WHtR & $4.11 \pm 0.48$ & $4.06 \pm 0.42$ & 0.257 \\
\hline TyG-WHpR & $7.43 \pm 0.65$ & $6.84 \pm 0.54$ & $<0.001$ \\
\hline Smoking & $228(62.81 \%)$ & $1(0.44 \%)$ & $<0.001$ \\
\hline Drinking & $210(57.85 \%)$ & $6(2.64 \%)$ & $<0.001$ \\
\hline Exercise & $78(21.49 \%)$ & $46(20.26 \%)$ & 0.030 \\
\hline
\end{tabular}

Data are presented as means \pm SD or number (percentage)

EH, essential hypertension; FPG,fasting plasma glucose; BMI, body mass index; ; WHpR, waist-to-hip ratio; WHtR, waist-toheight ratio; SBP,systolic blood pressure; DBP,diastolic blood pressure; TG, triglyceride; TC, total cholesterol; HDL-C, highdensity lipoprotein cholesterol; LDL-C, low-density lipoprotein cholesterol; TyG, triglyceride-glucose; TyG-WC, product of TyG and waist circumference; TyG-WHtR, product of TyG; TyG-WHpR, product of TyG and waist-to-hip ratio. 


\section{Logistic Regression Analyses For Tyg Index And Tyg-related Parameters With Mets Risk}

In the univariate logistic regression analysis, TyG index and TyG-related parameters were associated with Mets. This association persisted after adjustments for some Mets risk factors (age, gender, smoking, drinking, physical exercise, components of Mets). Before adjustment, TyG-WHtR presented the highest OR in all participants $(4.86,95 \%$ Cl: 2.98-7.95). After adjustment, TyG-WHtR presented the highest OR in all participants (5.63, 95\% Cl: 3.23-9.83). (Table 2).

Table 2. Logistic regression analyses for the relationship between various atherogenic parameters at baseline and incident Mets at follow-up in different models.

\begin{tabular}{|lllllll|}
\hline & Model 1 & & Model 2 & \multicolumn{3}{l|}{ Model 3 } \\
& OR(95 \% Cl) & P-value & OR $(95 \% \mathrm{Cl})$ & P-value & OR $(95 \% \mathrm{Cl})$ & P-value \\
\hline TyG index & $2.04(1.19,3.49)$ & 0.009 & $2.41(1.37,4.26)$ & 0.002 & $2.43(1.32,4.44)$ & 0.004 \\
\hline TyG-WC & $1.01(1.00,1.01)$ & $<0.001$ & $1.01(1.01,1.02)$ & $<0.001$ & $1.01(1.01,1.02)$ & $<0.001$ \\
\hline TyG-WHtR & $4.86(2.98,7.95)$ & $<0.001$ & $6.09(3.57,10.37)$ & $<0.001$ & $5.63(3.23,9.83)$ & $<0.001$ \\
\hline TyG-WHpR & $1.48(1.10,2.01)$ & 0.012 & $2.44(1.69,3.52)$ & $<0.001$ & $2.44(1.66,3.61)$ & $<0.001$ \\
\hline
\end{tabular}

Model 1: non-adjusted model;

Model 2: adjusted for age,gender,smoking,drinking,physical exercise;

Model 3: adjusted for age,gender,smoking,drinking,physical exercise and components of Mets (included EH,SBP,DBP and HDL-C).

Mets, metabolic syndrome; EH, essential hypertension; SBP,systolic blood pressure; DBP,diastolic blood pressure; HDL-C, high-density lipoprotein cholesterol; TyG, triglyceride-glucose; TyG-WC, product of TyG and waist circumference; TyG-WHtR, product of TyG; TyG-WHpR, product of TyG and waist-to-hip ratio

To determine the consistency of the relationship between TyG related parameters and risk of Mets, we conducted stratified analyses (Table 3). For non-adjusted model, TyG-related parameters significantly predicted Mets in both genders. TyG-WHtR was most strongly associated with Mets, the OR for Mets was 9.10 in males ( $P$ ख0.001) and 3.46 in females $(P=0.001)$. In Model 2, after adjusting for age, smoking, drinking and physical exercise, we found TyG-WHtR was the most strongly associated with Mets, the OR for Mets was 9.73 in males $(P \otimes 0.001)$ and $3.57(P=0.001)$ in females. After adjustments for components of Mets included HDL-C, SBP, DBP, and EH, only TyG-WHtR and TyG- WC significantly predicted Mets in both genders. The adjusted OR for TyG-WHtR in males was $9.14(P \otimes 0.001)$ compared with 3.18(P=0.005) in females. 
Table 3

Hazards ratios with 95\% confidence intervals for incident Mets increase in various atherogenic parameters in subgroups of gender.

\begin{tabular}{|c|c|c|c|c|c|c|c|c|c|}
\hline & \multicolumn{3}{|l|}{ Model 1} & \multicolumn{3}{|l|}{ Model 2} & \multicolumn{3}{|l|}{ Model 3} \\
\hline & $\mathrm{HR}(95 \% \mathrm{Cl})$ & $\begin{array}{l}P \\
\text { value }\end{array}$ & $\begin{array}{l}\text { P value } \\
\text { for } \\
\text { interaction }\end{array}$ & $\mathrm{HR}(95 \% \mathrm{Cl})$ & $\begin{array}{l}P \\
\text { value }\end{array}$ & $\begin{array}{l}\text { P value } \\
\text { for } \\
\text { interaction }\end{array}$ & $\mathrm{HR}(95 \% \mathrm{Cl})$ & $\begin{array}{l}\mathrm{P} \\
\text { value }\end{array}$ & $\begin{array}{l}\text { P value } \\
\text { for } \\
\text { interaction }\end{array}$ \\
\hline \multicolumn{10}{|l|}{$\begin{array}{l}\text { TyG } \\
\text { index }\end{array}$} \\
\hline Males & $\begin{array}{l}3.18(1.50 \\
6.73)\end{array}$ & 0.003 & 0.262 & $\begin{array}{l}3.25(1.51, \\
6.99)\end{array}$ & 0.003 & 0.251 & $\begin{array}{l}3.23(1.44 \\
7.28)\end{array}$ & 0.005 & 0.289 \\
\hline Females & $\begin{array}{l}1.68(0.73 \\
3.84)\end{array}$ & 0.220 & & $\begin{array}{l}1.67(0.72 \\
3.89)\end{array}$ & 0.231 & & $\begin{array}{l}1.69(0.69 \\
4.15)\end{array}$ & 0.251 & \\
\hline \multicolumn{10}{|l|}{ TyG-WC } \\
\hline Males & $\begin{array}{l}1.01(1.01 \\
1.02)\end{array}$ & $<.001$ & 0.338 & $\begin{array}{l}1.01 \\
1.02)\end{array}$ & $\dot{0} 001$ & 0.295 & $\begin{array}{l}1.01(1.01 \\
1.02)\end{array}$ & $\begin{array}{l}<.001 \\
0.00\end{array}$ & 0.194 \\
\hline Females & $\begin{array}{l}1.01(1.01 \\
1.02)\end{array}$ & $\begin{array}{l}< \\
0.001\end{array}$ & & $\begin{array}{l}1.01(1.01 \\
1.02)\end{array}$ & $\begin{array}{l}< \\
0.001\end{array}$ & & $\begin{array}{l}1.01(1.00 \\
1.02)\end{array}$ & $\begin{array}{l}<.001 \\
0.01\end{array}$ & \\
\hline \multicolumn{10}{|l|}{$\begin{array}{l}\text { TyG- } \\
\text { WHtR }\end{array}$} \\
\hline Males & $\begin{array}{l}9.10(4.35 \\
19.04)\end{array}$ & $<.001$ & 0.069 & $\begin{array}{l}9.73(4.55 \\
20.82)\end{array}$ & $<.001$ & 0.064 & $\begin{array}{l}9.14(4.16 \\
20.09)\end{array}$ & $\begin{array}{l}<.001 \\
0 .\end{array}$ & 0.062 \\
\hline Females & $\begin{array}{l}3.46(1.65 \\
7.26)\end{array}$ & 0.001 & & $\begin{array}{l}3.57(1.68 \\
7.59)\end{array}$ & 0.001 & & $\begin{array}{l}3.18(1.43 \\
7.08)\end{array}$ & 0.005 & \\
\hline \multicolumn{10}{|l|}{$\begin{array}{l}\text { TyG- } \\
\text { WHpR }\end{array}$} \\
\hline Males & $\begin{array}{l}3.14(1.92, \\
5.11)\end{array}$ & $\begin{array}{l}< \\
0.001\end{array}$ & 0.118 & $\begin{array}{l}3.12(1.90 \\
5.13)\end{array}$ & $\dot{0} 001$ & 0.127 & $\begin{array}{l}3.24(1.92 \\
5.47)\end{array}$ & $\begin{array}{l}<.001 \\
0 .\end{array}$ & 0.096 \\
\hline Females & $\begin{array}{l}1.75(1.01 \\
3.02)\end{array}$ & 0.044 & & $\begin{array}{l}1.76(1.01, \\
3.06)\end{array}$ & 0.047 & & $\begin{array}{l}1.67(0.92 \\
3.01)\end{array}$ & 0.089 & \\
\hline \multicolumn{10}{|c|}{ Model 1: non-adjusted model; } \\
\hline \multicolumn{10}{|c|}{ Model 2: adjusted for age,gender,smoking,drinking,physical exercise; } \\
\hline \multicolumn{10}{|c|}{$\begin{array}{l}\text { Model 3: adjusted for age,gender,smoking,drinking,physical exercise and components of Mets (included EH,SBP,DBP and } \\
\text { HDL-C). }\end{array}$} \\
\hline \multicolumn{10}{|c|}{$\begin{array}{l}\text { Mets, metabolic syndrome; EH, essential hypertension; SBP,systolic blood pressure; DBP,diastolic blood pressure; HDL-C, } \\
\text { high-density lipoprotein cholesterol; TyG, triglyceride-glucose; TyG-WC, product of TyG and waist circumference; TyG- } \\
\text { WHtR, product of TyG; TyG-WHpR, product of TyG and waist-to-hip ratio. }\end{array}$} \\
\hline
\end{tabular}

\section{Roc Curve Analyses For Tyg Index And Tyg-related Parameters With Mets Risk}

The ROC curve analyses are shown in Figure. 1A-C and the corresponding AUCs (95\% confidence interval, $\mathrm{Cl}$ ) in Table 4. Table 5 shows the pairwise comparison of the AUCs of TyG index, TyG-WC, TyG-WHpR, and TyG-WHtR for the detection of Mets. In all participants, TyG-WHtR shows the largest AUC for Mets detection (0.686) followed by TyG-WC (0.660), TyG- 
WHpR (0.564) and TyG-index (0.556) in that order. Analysis revealed that TyG-WHtR has the largest AUC in all participants, suggesting that it has the best discriminating power to identify Mets in comparison with other parameters.

Table 4

The areas under the receiver operating characteristic curve for each parameter for identifying Mets.

\begin{tabular}{|c|c|c|c|c|c|}
\hline Variable & AUC & $95 \% \mathrm{Cl}$ low & $95 \% \mathrm{Cl}$ upp & Specificity & Sensitivity \\
\hline \multicolumn{6}{|c|}{ All participants } \\
\hline TyG index & 0.5776 & 0.5111 & 0.6345 & 0.2687 & 0.8545 \\
\hline TyG-WC & 0.6771 & 0.6184 & 0.7203 & 0.2833 & 0.9364 \\
\hline TyG-WHpR & 0.5793 & 0.5194 & 0.6323 & 0.2812 & 0.8273 \\
\hline TyG-WHtR & 0.6967 & 0.6454 & 0.7484 & 0.3396 & 0.9364 \\
\hline \multicolumn{6}{|l|}{ Males } \\
\hline TyG index & 0.5981 & 0.5057 & 0.6905 & 0.8439 & 0.3673 \\
\hline TyG-WC & 0.7671 & 0.6973 & 0.8370 & 0.7134 & 0.7143 \\
\hline TyG-WHpR & 0.6960 & 0.6196 & 0.7724 & 0.7229 & 0.5714 \\
\hline TyG-WHtR & 0.7557 & 0.6850 & 0.8264 & 0.6783 & 0.7143 \\
\hline \multicolumn{6}{|l|}{ Females } \\
\hline TyG index & 0.5568 & 0.4772 & 0.6364 & 0.3012 & 0.8852 \\
\hline TyG-WC & 0.6956 & 0.6255 & 0.7657 & 0.3554 & 0.9672 \\
\hline TyG-WHpR & 0.5992 & 0.5201 & 0.6783 & 0.5000 & 0.6885 \\
\hline TyG-WHtR & 0.6493 & 0.5761 & 0.7225 & 0.2831 & 1.0000 \\
\hline
\end{tabular}

Table 5

Pairwise comparison of AUC of the different parameters.

\begin{tabular}{|c|c|c|c|}
\hline & All & males & females \\
\hline \multicolumn{4}{|l|}{ TyG_WHtR TyG_index } \\
\hline Difference between areas & 0.1307 & 0.1576 & 0.0925 \\
\hline $\mathrm{P}$ & 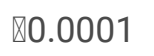 & 0.0002 & 0.0118 \\
\hline \multicolumn{4}{|l|}{ TyG_WHtR TyG_WC } \\
\hline Difference between areas & 0.0267 & 0.0114 & 0.0463 \\
\hline $\mathrm{P}$ & 0.0380 & 0.4305 & 0.0030 \\
\hline \multicolumn{4}{|l|}{ TyG_WHtR TyG_WHpR } \\
\hline Difference between areas & 0.1224 & 0.0654 & 0.0501 \\
\hline $\mathrm{P}$ & $<0.0001$ & 0.0090 & 0.0422 \\
\hline
\end{tabular}


Pairwise comparison of the AUCs showed that compared with other parameters, TyG-WHtR was the best in detecting Mets in all the participants (TyG-WHtR vs. TyG index, $\mathrm{P}<0.0001$; TyG-WHtR vs. TyG-WC, $\mathrm{P}=0.0380$; TyG-WHtR vs. TyG-WHpR, $\mathrm{P}<$ 0.0001). In males TyG-WHtR was as good as TyG-WC(TyG-WHtR vs. TyG index, $P=0.0002 ;$ TyG-WHtR vs. TyG-WC, $P=$ 0.4305; TyG-WHtR vs. TyG-WHpR, P = 0.0090) superior to other parameters in identifying Mets. In contrast, TyG-WC was better than TyG-WHtR (TyG-WHtR vs. TyG index, P = 0.0118; TyG-WHtR vs. TyG-WC, P = 0.0030; TyG-WHtR vs. TyG-WHpR, P = 0.0422) in detecting Mets in females.

\section{Discussion}

In this 15-year prospective follow-up study, we found that compared with the predictive ability of TyG index, TyG-WC and TyG-WHpR, TyG-WHtR with AUC of 0.683 was superior to other parameters for predicting Mets in all participants.

Furthermore, this study demonstrated that TyG-related markers that combine obesity markers with TyG index are superior to other parameters in identifying Mets in both genders. Further, TyG-WHtR showed the highest OR in all participants and both genders before and after adjustment.

A growing body of evidence indicates that IR may contribute to the progression of atherosclerotic cardiovascular disease and type 2 diabetes mellitus $[17,18]$. Because testing for insulin sensitivity is expensive, using the product of triglycerides and glucose as a surrogate marker to assess IR might help to minimize costs for clinical practice purpose[19]. TyG index, a product of triglycerides and glucose, was calculated as In (fasting triglycerides (mg dl-1) $\times$ fasting glucose $(\mathrm{mg} \mathrm{dl-1}) / 2)[20]$. A series of cohort and cross-sectional studies also confirmed that the TyG index can act as a better marker for predicting Mets[21-24]. However, a current study showed that the lipid accumulation product (LAP) is superior to TyG in predicting Mets in Koreans[25]. A Chinese study by Rui et al. also found that the LAP is better than TyG index for predicting the risk of Mets[26]. Both studies didn't compare with any TyG-related parameters. Consistent with our finding, Taiwo et al. found that TyG-WHtR is better than TyG index and other TyG-related parameters for predicting the risk of Mets in Nigerians[10]. The AUC of TyG index is 0.796 in identifying Mets in the cross-sectional study. The overall AUC for TyG index (0.556) in our study was even lower than other Chinese studies[22, 26, 27], but our cohort had a higher TG, lower FPG, similar TyG index. These may imply that there are not just ethnic differences, but regional differences among human subjects with regards to identifying Mets.

Consist with precious study[10], we carried out subgroup analysis by genders and found that TyG-WHtR as well as TyG-WC outperformed other indices in males at 15-year follow-up. Further, TyG-WHtR showed the highest OR before and after adjustment. Therefore, TyG-WHtR appears to be the best of all the parameters among all participants and males. Abdominal obesity includes both subcutaneous and visceral adipose tissue[28]. Visceral (intra-abdominal) fat is found to correlate more with cardiovascular risk, because they produce more fatty acids and secrete inflammatory cytokines and adipokines[29, 30]. Both WC and WHtR are markers of visceral adiposity[9]. Because WHtR corrected for height, it may be better than WC to predict Mets and cardiovascular risk[31,32]. The present study found that the accumulation of visceral adipose tissue accelerates the epigenetic age mostly mediated by TyG index in males[33]. In our study, it was found that the average age of the males was significantly older compared to the females. Therefore, we think that the TyG-WHtR was a significant predictor for Mets due to age-related metabolic dysfunctions occuring in adipose tissue in males.

In this study, all participants were representative of a limited geographical region (Chinese) and may not reflect the TyG index from the other countries. So that future large population-based multicenter studies are recommended. Moreover, Mets is just a complex multifactorial health problem, and it has limited practical utility as a diagnostic or management tool, but it is worthwhile to further elucidate the underlying pathways of the clustering of such a lot of risk factors.

\section{Conclusion}

The findings indicate that TyG-WHtR is superior to other parameters for predicting the risk of Mets in an urban Chinese population. The present study also reveals that TyG-related markers that combine obesity markers with TyG index are 
superior to other parameters in identifying Mets in both genders.

\section{Abbreviations}

Mets:metabolic syndrome;IR:insulin resistance;TyG index:triglyceride-glucose index; FPG:fasting plasma

glucose;TG:triglycerides;TyG-WC:TyG-waist circumference;TyG-WHtR:TyG-waist-to height ratio;TyG-WHpR:TyG-waist-to-hip ratio; $\mathrm{BP}$ :systolic blood pressure;BMl:body mass index;FPG:fasting plasma glucose; TG, triglyceride; TC, total cholesterol; HDL-C, high-density lipoprotein cholesterol; LDL-C, low-density lipoprotein cholesterol;EH, essential hypertension; ROC:receiver operating characteristic;AUC:area under the curve;LAP:lipid accumulation product

\section{Declarations}

\section{Authors'contributions}

All authors read and approved the final manuscript.

Acknowledgements Not applicable

Funding There was no funding for this work.

Conflict of interests The authors declare that they have no competing interests.

\section{Availability of data and materials}

All data generated or analyzed during this study are included in this published article.

\section{Ethics approval and consent to participate}

Not applicable.

\section{Consent for publication}

Not applicable.

\section{Competing interests}

The authors declare that they have no competing interests.

\section{References}

1. Alberti KG, Eckel RH, Grundy SM, Zimmet PZ, Cleeman JI, Donato KA, Fruchart JC, James WP, Loria CM, Smith SJ. Harmonizing the metabolic syndrome: a joint interim statement of the International Diabetes Federation Task Force on Epidemiology and Prevention; National Heart, Lung, and Blood Institute; American Heart Association; World Heart Federation; International Atherosclerosis Society; and International Association for the Study of Obesity. Circulation. 2009; 120(16):1640-1645.

2. Chen X, He C, Ma Y, Yang Y, Liu F, Ma X, Li X, Xie X, Chen B. Association of metabolic syndrome with various anthropometric and atherogenic parameters in the Kazakh population in China. Lipids Health Dis. 2016;15(1):166.

3. Cerf ME. Beta cell dysfunction and insulin resistance. Front Endocrinol (Lausanne). 2013;4:37.

4. Abbasi F, Reaven GM. Comparison of two methods using plasma triglyceride concentration as a surrogate estimate of insulin action in nondiabetic subjects: triglycerides $\mathrm{x}$ glucose versus triglyceride/high-density lipoprotein cholesterol. 
Metabolism. 2011;60(12):1673-6.

5. Lee DY, Lee ES, Kim JH, Park SE, Park CY, Oh KW, Park SW, Rhee EJ, Lee WY. Predictive Value of Triglyceride Glucose Index for the Risk of Incident Diabetes: A 4-Year Retrospective Longitudinal Study. PLoS ONE. 2016;11(9):e163465.

6. Zheng R, Mao Y. Triglyceride and glucose (TyG) index as a predictor of incident hypertension: a 9-year longitudinal population-based study. Lipids Health Dis. 2017;16(1):175.

7. Sanchez-Inigo L, Navarro-Gonzalez D, Fernandez-Montero A, Pastrana-Delgado J, Martinez JA. The TyG index may predict the development of cardiovascular events. Eur J Clin Invest. 2016;46(2):189-97.

8. Zhang S, Du T, Zhang J, Lu H, Lin X, Xie J, Yang Y, Yu X. The triglyceride and glucose index (TyG) is an effective biomarker to identify nonalcoholic fatty liver disease. Lipids Health Dis. 2017;16(1):15.

9. Lim J, Kim J, Koo SH, Kwon GC. Comparison of triglyceride glucose index, and related parameters to predict insulin resistance in Korean adults: An analysis of the 2007-2010 Korean National Health and Nutrition Examination Survey. PLoS ONE. 2019;14(3):e212963.

10. Raimi TH, Dele-Ojo BF, Dada SA, Fadare JO, Ajayi DD, Ajayi EA, Ajayi OA. Triglyceride-Glucose Index and Related Parameters Predicted Metabolic Syndrome in Nigerians. Metab Syndr Relat Disord. 2021;19(2):76-82.

11. The World Health Organization MONICA Project. (monitoring trends and determinants in cardiovascular disease): a major international collaboration. WHO MONICA Project Principal Investigators. J Clin Epidemiol. 1988;41(2):105-14.

12. Ren J, Grundy SM, Liu J, Wang W, Wang M, Sun J, Liu J, Li Y, Wu Z, Zhao D. Long-term coronary heart disease risk associated with very-low-density lipoprotein cholesterol in Chinese: the results of a 15-Year Chinese Multi-Provincial Cohort Study (CMCS). Atherosclerosis. 2010;211(1):327-32.

13. Liu J, Hong Y, D'Agostino RS, Wu Z, Wang W, Sun J, Wilson PW, Kannel WB, Zhao D. Predictive value for the Chinese population of the Framingham CHD risk assessment tool compared with the Chinese Multi-Provincial Cohort Study. JAMA. 2004;291(21):2591-9.

14. Zhang X, Zhang X, Li X, Feng J, Chen X. Association of metabolic syndrome with atherogenic index of plasma in an urban Chinese population: A 15-year prospective study. Nutr Metab Cardiovasc Dis. 2019;29(11):1214-9.

15. Wang S, Liu K, Zhang X, Meng Q, Wang Y, Wan S, Chen X. Elevated resting heart rate predisposes metabolic syndrome in women rather than in men: a 15-year prospective study. BMC Cardiovasc Disord. 2015;15:110.

16. Grundy SM, Cleeman JI, Daniels SR, Donato KA, Eckel RH, Franklin BA, Gordon DJ, Krauss RM, Savage PJ, Smith SJ, et al. Diagnosis and management of the metabolic syndrome: an American Heart Association/National Heart, Lung, and Blood Institute Scientific Statement. Circulation. 2005;112(17):2735-52.

17. Cheng YH, Tsao YC, Tzeng IS, Chuang HH, Li WC, Tung TH, Chen JY. Body mass index and waist circumference are better predictors of insulin resistance than total body fat percentage in middle-aged and elderly Taiwanese. Med (Baltim). 2017;96(39):e8126.

18. Meigs JB, Wilson PW, Fox CS, Vasan RS, Nathan DM, Sullivan LM, D'Agostino RB. Body mass index, metabolic syndrome, and risk of type 2 diabetes or cardiovascular disease. J Clin Endocrinol Metab. 2006;91(8):2906-12.

19. Du T, Yuan G, Zhang M, Zhou X, Sun X, Yu X. Clinical usefulness of lipid ratios, visceral adiposity indicators, and the triglycerides and glucose index as risk markers of insulin resistance. Cardiovasc Diabetol. 2014;13:146.

20. Simental-Mendia LE, Rodriguez-Moran M, Guerrero-Romero F. The product of fasting glucose and triglycerides as surrogate for identifying insulin resistance in apparently healthy subjects. Metab Syndr Relat Disord. 2008;6(4):299304.

21. Khan SH, Sobia F, Niazi NK, Manzoor SM, Fazal N, Ahmad F. Metabolic clustering of risk factors: evaluation of Triglyceride-glucose index (TyG index) for evaluation of insulin resistance. Diabetol Metab Syndr. 2018;10:74.

22. Yu X, Wang L, Zhang W, Ming J, Jia A, Xu S, Li Q, Ji Q. Fasting triglycerides and glucose index is more suitable for the identification of metabolically unhealthy individuals in the Chinese adult population: A nationwide study. J Diabetes Investig. 2019;10(4):1050-8. 
23. Lee SH, Han K, Yang HK, Kim HS, Cho JH, Kwon HS, Park YM, Cha BY, Yoon KH. A novel criterion for identifying metabolically obese but normal weight individuals using the product of triglycerides and glucose. Nutr Diabetes. 2015;5:e149.

24. Son DH, Lee HS, Lee YJ, Lee JH, Han JH. Comparison of triglyceride-glucose index and HOMA-IR for predicting prevalence and incidence of metabolic syndrome. Nutr Metab Cardiovasc Dis. 2021.

25. Shin KA, Kim YJ. Usefulness Of Surrogate Markers Of Body Fat Distribution For Predicting Metabolic Syndrome In Middle-Aged And Older Korean Populations. Diabetes Metab Syndr Obes. 2019;12:2251-9.

26. Li R, Li Q, Cui M, Yin Z, Li L, Zhong T, Huo Y, Xie P. Clinical surrogate markers for predicting metabolic syndrome in middle-aged and elderly Chinese. J Diabetes Investig. 2018;9(2):411-8.

27. Lin HY, Zhang XJ, Liu YM, Geng LY, Guan LY, Li XH. Comparison of the triglyceride glucose index and blood leukocyte indices as predictors of metabolic syndrome in healthy Chinese population. Sci Rep. 2021;11(1):10036.

28. Mclaughlin T, Lamendola C, Liu A, Abbasi F. Preferential fat deposition in subcutaneous versus visceral depots is associated with insulin sensitivity. J Clin Endocrinol Metab. 2011;96(11):E1756-60.

29. Boden G, Shulman GI. Free fatty acids in obesity and type 2 diabetes: defining their role in the development of insulin resistance and beta-cell dysfunction. Eur J Clin Invest. 2002;32(Suppl 3):14-23.

30. Kwon H, Pessin JE. Adipokines mediate inflammation and insulin resistance. Front Endocrinol (Lausanne). $2013 ; 4: 71$.

31. Yang H, Xin Z, Feng JP, Yang JK. Waist-to-height ratio is better than body mass index and waist circumference as a screening criterion for metabolic syndrome in Han Chinese adults. Med (Baltim). 2017;96(39):e8192.

32. Zhang ZQ, Deng J, He LP, Ling WH, Su YX, Chen YM. Comparison of various anthropometric and body fat indices in identifying cardiometabolic disturbances in Chinese men and women. PLoS ONE. 2013;8(8):e70893.

33. Arpon A, Milagro FI, Santos JL, Garcia-Granero M, Riezu-Boj JI, Martinez JA. Interaction Among Sex, Aging, and Epigenetic Processes Concerning Visceral Fat, Insulin Resistance, and Dyslipidaemia. Front Endocrinol (Lausanne). 2019;10:496.

\section{Figures}



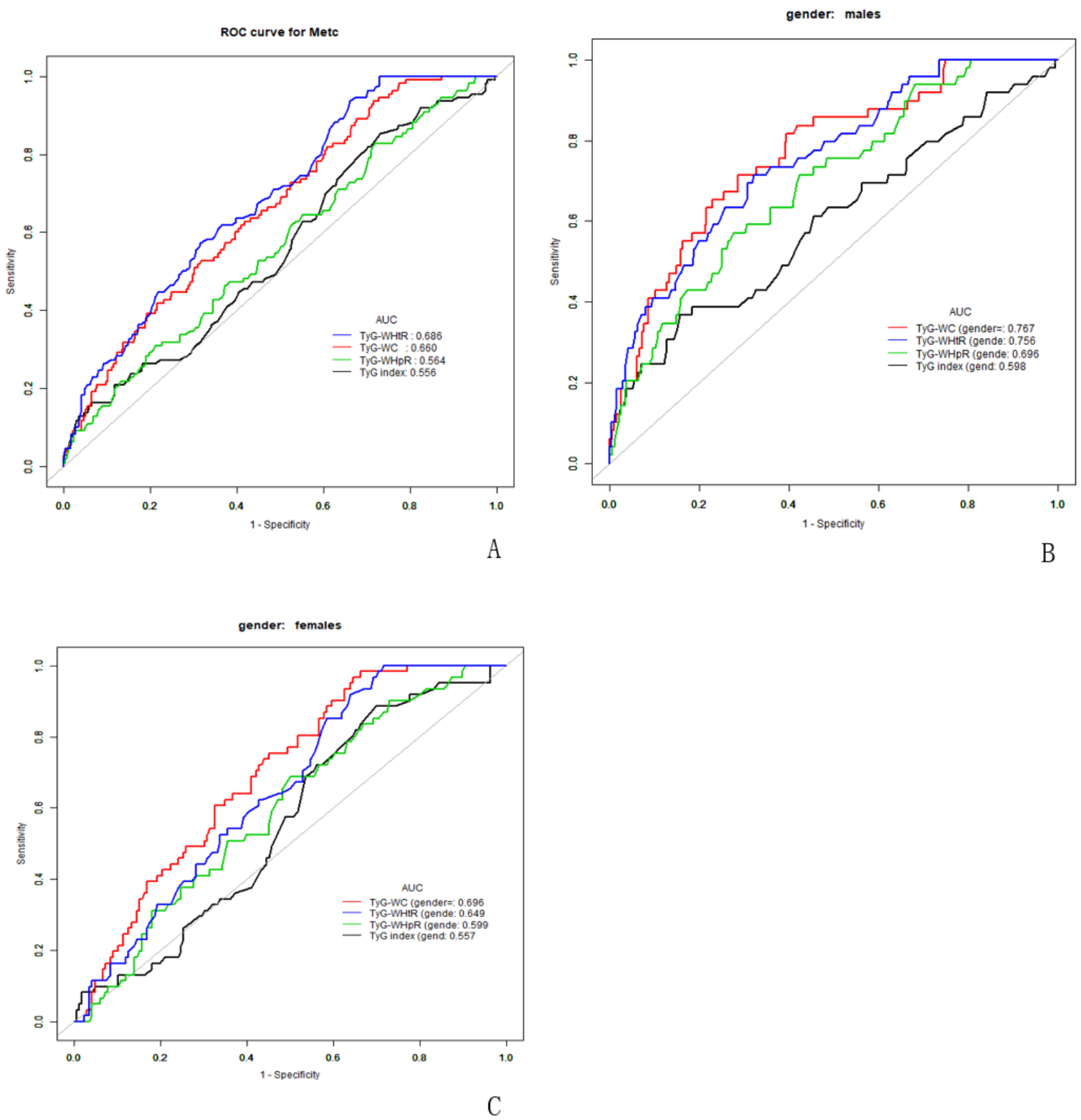

\section{Figure 1}

ROC curves for the parameters for identifying Mets. (A) ROC curve for each parameter for identifying Mets in all participants. (B) ROC curve for Mets each parameter for identifying Mets in males. (C) ROC curve for each parameter for identifying Mets in females.

Mets, metabolic syndrome; ROC, receiver operating characteristic; TyG, triglyceride-glucose; TyG-WC, product of TyG and waist circumference; TyG-WHtR, product of TyG and waist-to-height ratio; TyG-WHpR, product of TyG and waist-to-hip ratio. 